\title{
Exploring New Intuitive Interfaces for a Natural Design Process
}

\author{
Prieto P.A \\ Universidad Técnica Federico Santa María \\ Av. España 1680. Valparaíso. Chile \\ pablo.prieto@usm.cl
}

Given the key role that digital systems have in modern manufacturing processes nowadays, the creative part of the design process usually goes through the following stages: Sketching > Physical Modeling > Digital modeling > Digital simulation > Manufacturing. When moving through these stages two fundamental requirements should be met: 1) the transference of new ideas from the designer mind to the physical/digital representation of them should be smooth and 2) the accuracy of the data while moving through the different stages should be ensured -especially when transiting from the physical to the digital modeling.

Meeting such requirements is still a challenge, mainly of interface nature. On one hand, current available tools to represent 3D objects rather hamper the smoothness of the idea-model transition process: quick hand sketching is limited by its 2D nature, which has infinite 3D interpretations; 3D soft modeling is constrained by its updating speed, especially in the beginning of the design process where the original idea is constantly modified and refined; 3D digital modeling is not only constrained by its updating speed but also by the functionalities of the software tools available (normally unable of accurately representing free-form surfaces). On the other hand, the transfer of the model data from one stage to another still lacks accuracy: in spite of many efforts in developing software tools for 3D interpretation of sketches, the transfer from 2D to 3D data still misses or misinterpret many features of the original design intent, whilst the transfer of free-form surfaces from the soft model to the 3D digital representation is still a time consuming task.

The main goal of this ongoing work is solving the above mentioned problems, namely: the limitations of bi-dimensional nature of sketching, the speed constrains imposed by the transition between the physical and the digital model and the limitations of current CAD systems to accurately represent free-form surfaces. To do so, a new intuitive interface which uses the functionalities of already mature multi-touch screens, digital video processing and augmented reality is proposed.

A multi-touch screen bridges the gap between the scale difference between the computer screen and the surface area in which the mouse moves, allowing a more direct and smooth interaction between the user and the sketch being produced. In this work a multi-touch screen will be used as a sketch surface of the parallel projection of the object the designer has in mind (in this first stage, the objects should have very simple shapes, like a cylinder). The figures sketched in the multi-touch screen are saved in a CAD program and used as a base for 3D modeling which will be based on human gesture and video processing. For the designer to be able to actually "see" the ongoing $3 \mathrm{~d}$ modelling process, augmented reality will be used. As a normal computer screen would inherit the limitations of 2D representations, instead of a 2D screen, a 3D screen is planned to be used.

The system is intended as a first step in the way of facilitating the different transitions between the physical and digital models (2d sketching $>3 \mathrm{~d}$ digital sketching or Soft models $>$ Digital models > real prototypes) in the creative part of the design process. Through the integration of emerging technologies, the system allows the integration of previous approaches which have focused more on testing of ergonomic or visual features of models rather than on on-line geometric modelling.

It is expected that the future prototype system provides engineers and designers with a very intuitive and real-world interface which assists them in the conceptual stages of the design process. 